\title{
Correction: Molecular analysis of subtilase cytotoxin genes of food-borne Shiga toxin-producing Escherichia coli reveals a new allelic subAB variant
}

Joschua Funk, Helen Stoeber, Elisabeth Hauser and Herbert Schmidt ${ }^{*}$

\section{Correction}

After publication of this work [1], we noticed that the sequences of primers subA-L and subAB5'-OEP in Table two (Table 1 here) are not correct. We therefore provide the correct primer sequences in a new Table two (Table 1 here).

Table 1 Designations, targets, and positions of primers for PCR analysis and Southern blot hybridization

\begin{tabular}{|c|c|c|c|c|}
\hline Primer & Target & Sequence $\left(5^{\prime}-3^{\prime}\right)$ & GenBank accession number & Reference \\
\hline saaDF & saa & 5'-CGT GAT GAA CAG GCT ATT GC-3' & AF399919 & {$[26]$} \\
\hline saaDR & saa & 5'-ATG GAC ATG CCT GTG GCA AC-3' & AF399919 & {$[26]$} \\
\hline subAB-V-for & $s u b A B$ & 5'-CTT CCC TCA TTG CCT CAC G-3' & AY258503 & This study \\
\hline subAB-V-rev & $s u b A B$ & 5'-GGC TGG CCT GTT GTG TAA A-3' & AY258503 & This study \\
\hline tia_lo & tia & 5'-TCC ATG CGA AGT TGT TAT CA-3' & U20318 & {$[15]$} \\
\hline tia_sense & tia & 5'-TTC TCT TाT TAC CCT GCT TाT TGC-3' & FJ664545 & {$[15]$} \\
\hline subAB-for5 & $s u b A B_{1}$ & 5'-CGT ATC TGC GCC ATA TCC TG-3' & AY258503 & This study \\
\hline subAB-rev5 & $s u b A B_{1}$ & 5'-CTG TTC CGA GCA GCC ATA TC-3' & AY258503 & This study \\
\hline subAB3'tia & $\operatorname{sub} A B_{2-1}$ & 5'-ACT GGC TGT TCT AAC CG-3' & AEZO02000028.1 & This study \\
\hline subA_out & $\operatorname{subA}_{2-2}$ & $5^{\prime}-$ GAA TCA ACA ACA GAT ACG AC-3' & AEZO02000020.1 & This study \\
\hline subA-L & Linker $^{a}$ & $5^{\prime}-\mathrm{GGG}$ AGG ATT AAC CAT CG-3' & AEZO02000020.1 & This study \\
\hline subAB5'OEP & $s u b A B_{2-2}$ & 5'-ATG AAT GAG AGC ATC CCT-3' & AEZO02000020.1 & This study \\
\hline subAB2-3'out & $s u b A B_{2-2}$ & 5'-AGG TCG GCT CAG TGT TC-3' & AEZO02000020.1 & This study \\
\hline
\end{tabular}

${ }^{\mathrm{a}}$ Intergenic linker between the OEP-locus and $s u b A_{2-2}$.

Received: 16 January 2014 Accepted: 16 January 2014

Published: 10 February 2014

\section{Reference}

1. Funk J, Stoeber H, Hauser E, Schmidt H: Molecular analysis of subtilase cytotoxin genes of food-borne shiga toxin-producing escherichia coli reveals a new allelic subAB variant. BMC Microbiol 2013, 13:230.

\section{doi:10.1186/1471-2180-14-32}

Cite this article as: Funk et al:: Correction: Molecular analysis of subtilase cytotoxin genes of food-borne Shiga toxin-producing Escherichia coli reveals a new allelic subAB variant. BMC Microbiology 2014 14:32.

\section{* Correspondence: herbert.schmidt@uni-hohenheim.de}

Department of Food Microbiology, Institute of Food Science and

Biotechnology, University of Hohenheim, Garbenstraße 28, D-70599 Stuttgart, Germany 